\title{
Coexisting Attractors in a New Smooth Inductorless Chua's Circuit
}

\author{
Lei $\mathrm{Zhu}^{1,2}$ and Minghai Pan ${ }^{1, *}$ \\ ${ }^{1}$ College of Electronic and Information Engineering, Nanjing University of Aeronautics and Astronautics, Nanjing, China \\ ${ }^{2}$ College of Electrical and Information Engineering, Jiangsu University of Technology, Changzhou, China \\ *Corresponding author
}

\begin{abstract}
The inductorless design method can facilitate experiment debugging and even engineering applications for various circuits. By introducing active diode pair as Chua's diode, a new smooth inductorless Chua's circuit is proposed based on a band pass filter with second order Sallen-Key topology. The dynamical analyses for this circuit are numerically carried out by use of bifurcation diagrams, Lyapunov exponent spectra and phase portraits. The results of numerical simulations and further circuit simulations reveal that this new circuit has rich dynamic behaviors of coexisting periodic attractors, coexisting single-scroll chaotic attractors, double-scroll chaotic attractor, periodic windows, boundary crisis and coexisting period-doubling bifurcation modes. This new circuit model and the corresponding research results enrich our understanding to Chua's circuit.
\end{abstract}

Keywords-smooth inductorless Chua's circuit; active diode pair; coexisting attractors; coexisting period-doubling bifurcation modes

\section{INTRODUCTION}

Chua's circuit [1] is an important nonlinear circuit which is composed of one inductor, two capacitors, one resistor and one Chua's diode. Because of the troubles for making and applying inductance, various inductorless Chua's circuits get a lot of attention by researchers. In [2], inductorless Chua's circuits are divided into two categories from the similarity to the traditional Chua's circuit. In this article, a new inductorless Chua's circuit that belongs to the model of FTICC [2] is presented based on a second order Sallen-Key BPF. Unlike the inductorless Chua's circuit in [2], an active diode pair [4] is introduced in the new circuit as a Chua's diode. Because of the hyperbolic sine nonlinearity in active diode pair, the new circuit is smooth, not piecewise-linear.

\section{NEW SMOOTH INDUCTORLESS CHUA`s CiRCUIT}

The new smooth inductorless Chua's circuit is drawn in FIGURE I, which is composed of three parts with different colors for connected lines. Here the partial circuit on the left of port 22' is a second order Sallen-Key band pass filter (BPF) [3], and the partial circuit between port 11' and $22^{\prime}$ is a resistance capacitance coupled network, and the partial circuit on the right of port 11' is a smooth Chua's diode formed by active diode pair [4]. According to the classification framework of inductorless Chua's circuits proposed in [2], this new circuit belongs to the FTICC model [2].

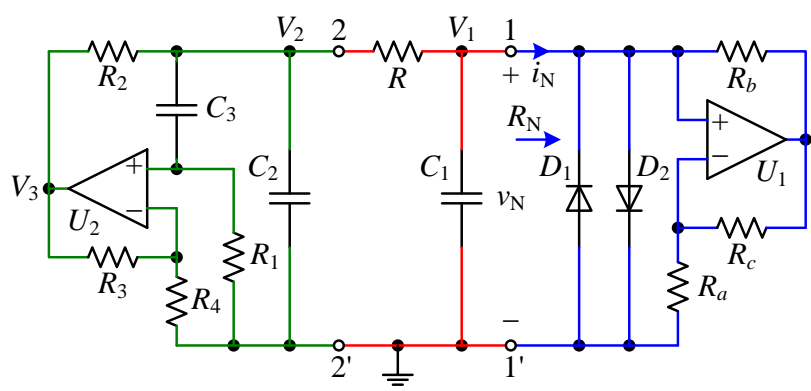

FIGURE I. SCHEMATIC OF THE NEW SMOOTH INDUCTORLESS CHUA'S CIRCUIT

The active diode pair [4] $R_{\mathrm{N}}$ connected by blue lines in FIGURE $I$ is composed of diodes $D_{1}, D_{2}$ and negative resistance $-R_{a}$. The volt-ampere characteristic of $R_{\mathrm{N}}$ can be expressed as

$$
i_{\mathrm{N}}=h\left(v_{\mathrm{N}}\right)=\frac{-v_{\mathrm{N}}}{R_{a}}+2 I_{S} \sinh \left(\frac{v_{\mathrm{N}}}{n V_{T}}\right)
$$

Here $I_{S}, n$, and $V_{T}$ denote the reverse saturation current, emission factor, and thermal voltage for the corresponding diode separately. Based on Kirchhoff's laws, a group of differential equations for describing this new smooth inductorless Chua's circuit can be obtained. Under a certain condition of $C_{2}=C_{3}$, the differential equations are summarized as

$$
\left\{\begin{array}{l}
C_{1} \frac{\mathrm{d} V_{1}}{\mathrm{~d} t}=-\frac{1}{R} V_{1}+\frac{1}{R} V_{2}-h\left(V_{1}\right) \\
C_{2} \frac{\mathrm{d} V_{2}}{\mathrm{~d} t}=\frac{1}{R} V_{1}-\left(\frac{1}{R}+\frac{1}{R_{2}}\right) V_{2}+\left(-\frac{1}{(1+k) R_{1}}+\frac{1}{R_{2}}\right) V_{3} \\
C_{3} \frac{\mathrm{d} V_{3}}{\mathrm{~d} t}=\frac{1+k}{R} V_{1}-(1+k)\left(\frac{1}{R}+\frac{1}{R_{2}}\right) V_{2}+\left(-\frac{2}{R_{1}}+\frac{1+k}{R_{2}}\right) V_{3}
\end{array}\right.
$$


where $k=R_{3} / R_{4}$, and the nonlinear function $h\left(V_{1}\right)$ is given by (1).

By numerical simulations and circuit simulations, a set of typic circuit parameters can be given as $C_{1}=20 \mathrm{nF}, C_{2}=C_{3}=$ $100 \mathrm{nF}, R_{a}=1.5 \mathrm{k} \Omega, R_{b}=R_{c}=0.5 \mathrm{k} \Omega, R=3.55 \mathrm{k} \Omega, R_{1}=R_{2}=$ $0.5 \mathrm{k} \Omega, R_{3}=2.2 \mathrm{k} \Omega$ and $R_{4}=1 \mathrm{k} \Omega$. For the diodes and op amps, the models of $1 \mathrm{~N} 4148$ and AD711AQ are chosen respectively. Consequently, the constants of $I_{S}=6.8913 \mathrm{nA}, n=1.8268$ and $V_{T}=25 \mathrm{mV}$ can be achieved [4]. Under these conditions, a double-scroll chaotic attractor is triggered by the initial values $\left(V_{1}(0), V_{2}(0), V_{3}(0)\right)=(1 \mathrm{mV}, 0,0)$, as shown in FIGURE II.

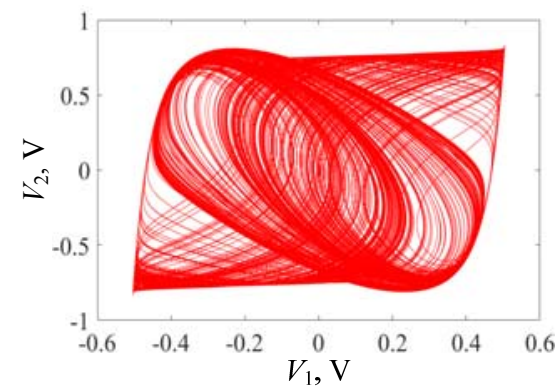

(a)

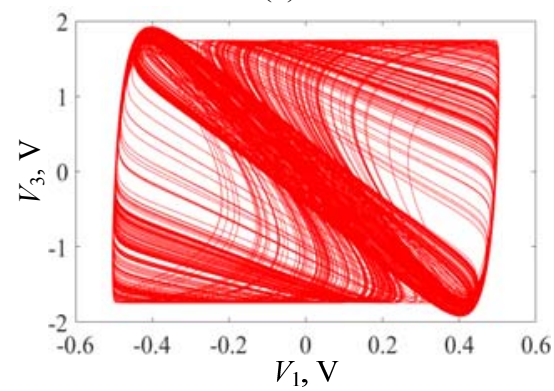

(b)

FIGURE II. THE DOUBLE-SCROLL CHAOTIC ATTRACTOR FOR THE NEW SMOOTH INDUCTORLESS CHUA'S CIRCUIT. (A) V1 - V2 PLANE; (B) V1 - V3 PLANE.

\section{DYNAMICAL ANALYSES}

By letting the right of (2) equal to 0 , the three equilibrium points for this circuit can be expressed as

$$
\left\{\begin{array}{l}
Q_{0}=(0,0,0) \\
Q_{ \pm}=\left( \pm \mu, \mp \frac{R_{2}}{R_{2}+R} \mu, \mp \frac{2(1+k) R_{1} R_{2}}{\left[(1+k) R_{1}-R_{2}\right] R} \mu\right)
\end{array}\right.
$$

where $\mu$ is the positive real root of the nonlinear equation

$$
\left[\frac{1}{R_{a}}-\frac{2 R_{2}+R}{R\left(R_{2}+R\right)}\right] \mu-2 I_{S} \sinh \left(\frac{\mu}{n V_{T}}\right)=0
$$

For the typic circuit parameters, the equilibrium points can be numerically achieved based on (3) and (4), as listed by

$$
\left\{\begin{array}{l}
Q_{0}=(0,0,0) \\
Q_{ \pm}=( \pm 0.459352, \mp 0.056710, \mp 0.344479)
\end{array}\right.
$$

Further, the eigenvalues corresponding to the three equilibrium points in (5) can be calculated as

$$
\begin{aligned}
& Q_{0}: \lambda_{1}=21094.187, \quad \lambda_{2,3}=-338.67561 \pm \mathrm{j} 21297.705 \\
& Q_{ \pm}: \lambda_{1,2}=698.9601 \pm \mathrm{j} 21217.305, \quad \lambda_{3}=-157084.06
\end{aligned}
$$

Hence, these three equilibrium points are all unstable. According to the categories of attractors in [5], the doublescroll chaotic attractor under the typic circuit parameters is selfexcited, not hidden.

In order to observe the dynamical behaviors in the new circuit more directly, the bifurcation diagrams and Lyapunov exponent spectra about parameter $R$ are drawn based on the typic circuit parameters, as shown in FIGURE III. The bifurcation diagrams are simulated by the local maximum of $V_{1}$, where the blue trajectories correspond to the initial values $\left(V_{1}(0), V_{2}(0), V_{3}(0)\right)=(-1 \mathrm{mV}, 0,0)$ and the red trajectories correspond to the initial values $\left(V_{1}(0), V_{2}(0), V_{3}(0)\right)=(1 \mathrm{mV}, 0$, $0)$. Obviously, with the increase of the value for resistance $R$, the circuit state evolves into chaos through coexisting perioddoubling bifurcation modes. A boundary crisis triggers the state transition from single-scroll chaotic attractor to double-scroll one further. Therefore, the new smooth inductorless Chua's circuit has the similar dynamical behaviors with the traditional Chua's circuit [1]. The Lyapunov exponents in FIGURE III (b) are simulated by Wolf's algorithm [6], where the third negative exponential curve is omitted for clarity. It can be found that the Lyapunov exponent spectra remain consistent with bifurcation diagrams. Moreover, various periodic windows with different sizes exist in the chaotic interval.

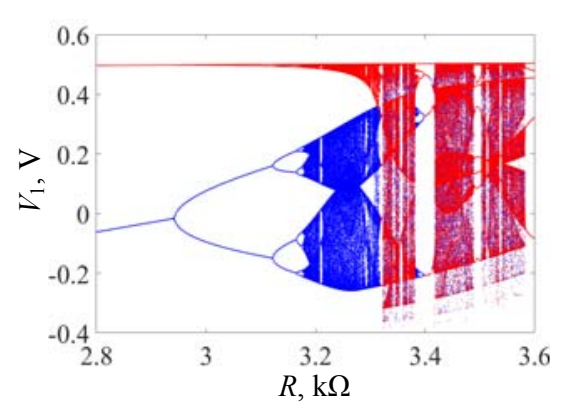

(a) 


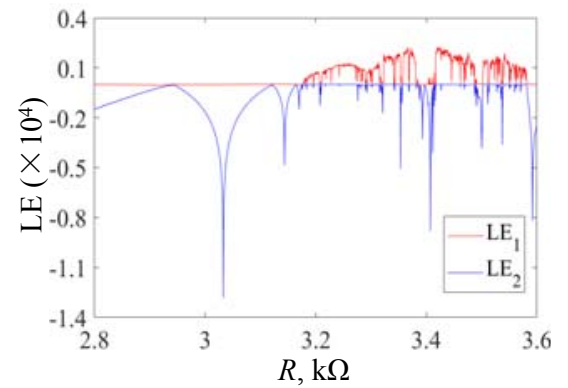

(b)

FIGURE III. DYNAMICAL BEHAVIORS ABOUT R. (A) BIFURCATION DIAGRAMS; (B) LYAPUNOV EXPONENT SPECTRA.

By letting $R$ equals to $2.9 \mathrm{k} \Omega, 3.1 \mathrm{k} \Omega, 3.15 \mathrm{k} \Omega$ and $3.25 \mathrm{k} \Omega$ respectively, the coexisting oscillation behaviors of period-1, period-2, period-4 and chaos can be discovered accordingly, as shown in FIGURE IV. Here these initial states corresponding to two colors are consistent with those ones in FIGURE III (a).

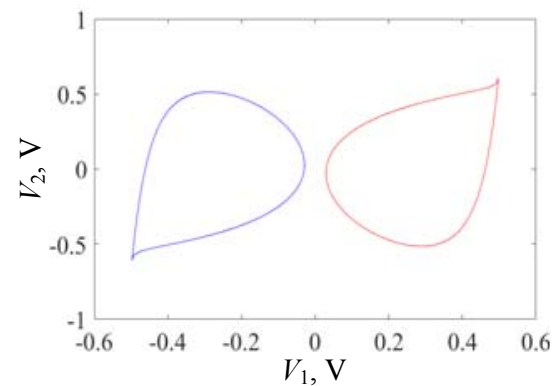

(a)

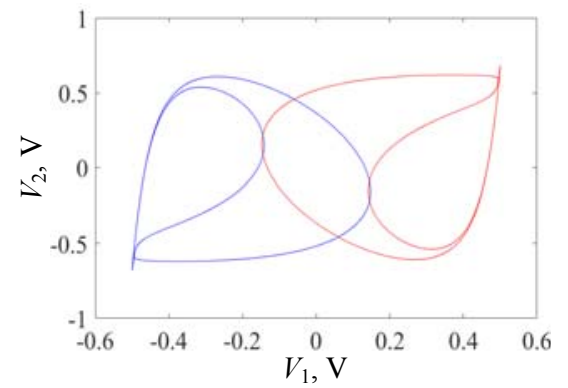

(b)

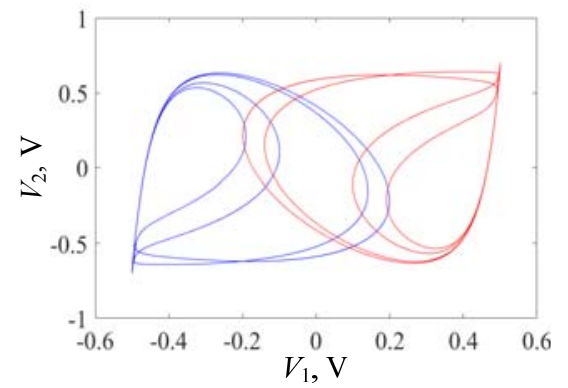

(c)

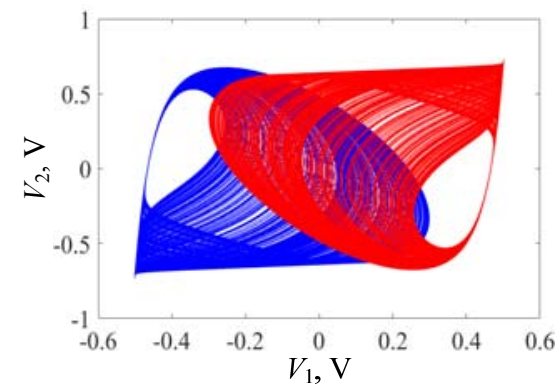

(d)

FIGURE IV. THE PHASE PORTRAITS IN V1 - V2 PLANE WITH DIFFERENT R. (A) $\mathrm{R}=2.9 \mathrm{~K} \Omega$; (B) $\mathrm{R}=3.1 \mathrm{~K} \Omega$; (C) $\mathrm{R}=3.15 \mathrm{~K} \Omega$; (D) $\mathrm{R}=3.25 \mathrm{~K} \Omega$.

\section{Circuit Simulations}

The above numerical simulations are performed by the ode23 algorithm in MATLAB software. In order to better verify the above analysis, a series of circuit simulation results are provided below. Based on Multisim 12.0 software, the simulation results in the typic circuit parameters are presented by FIGURE V, where the initial state of capacitance $C_{1}$ is set to $1 \mathrm{mV}$. Obviously this double-scroll chaotic attractor achieved by circuit simulation is consistent with the one in FIGURE II basically, except the corresponding oscillation amplitudes for three state variables increase to some extent.

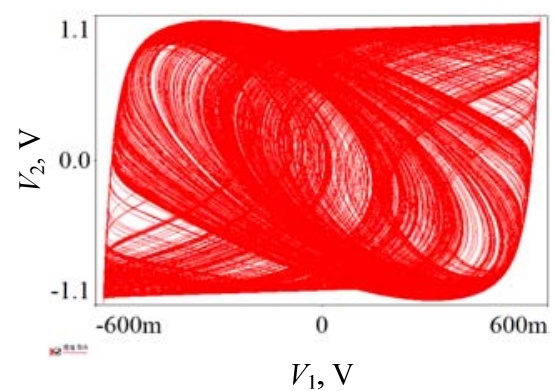

(a)

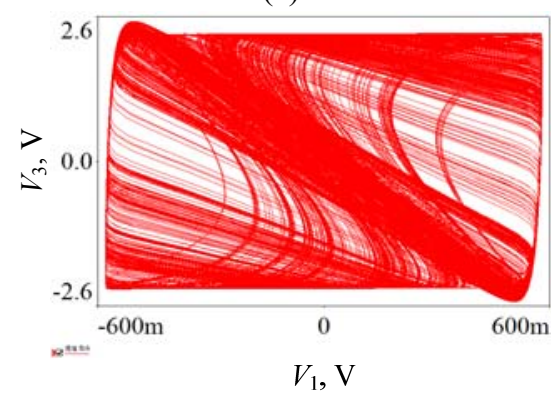

(b)

FIGURE V. THE DOUBLE-SCROLL CHAOTIC ATTRACTOR ACHIEVED BY CIRCUIT SIMULATION. (A) V1 - V2 PLANE; (B) V1 - V3 PLANE.

Similarly, by letting $R$ equals to $2.9 \mathrm{k} \Omega, 3.1 \mathrm{k} \Omega, 3.15 \mathrm{k} \Omega$ and $3.25 \mathrm{k} \Omega$ respectively, the coexisting attractors of period-1, period-2, period-4 and chaos can be achieved by circuit simulations accordingly, as given in FIGURE VI. It's worth 


\section{CONCLUSIONS}

noting that the initial state of capacitance $C_{1}$ is set to $-1 \mathrm{mV}$ for blue phase portraits and $1 \mathrm{mV}$ for red ones. These circuit simulation results are consistent with those numerical results in FIGURE IV except the little increases of oscillation amplitudes, thereby further verifying the coexisting period-doubling bifurcation behaviors in the new smooth inductorless Chua's circuit.

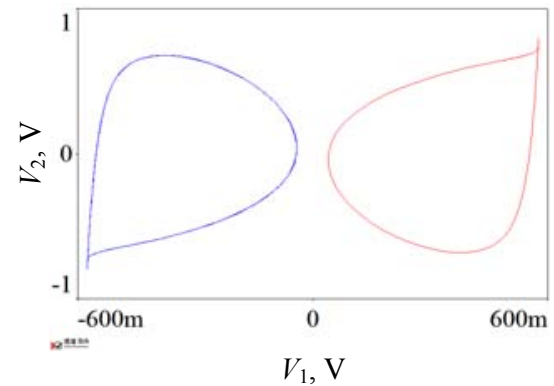

(a)

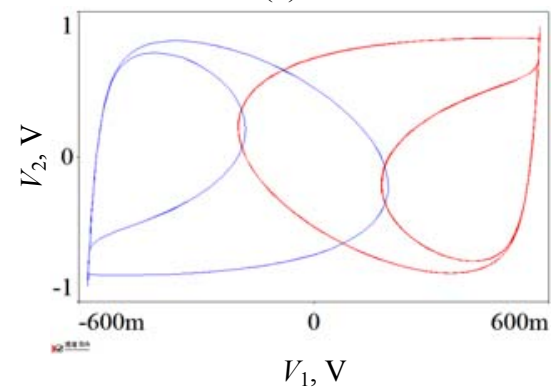

(b)

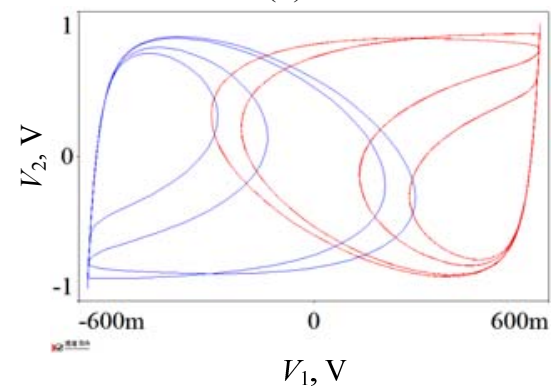

(c)

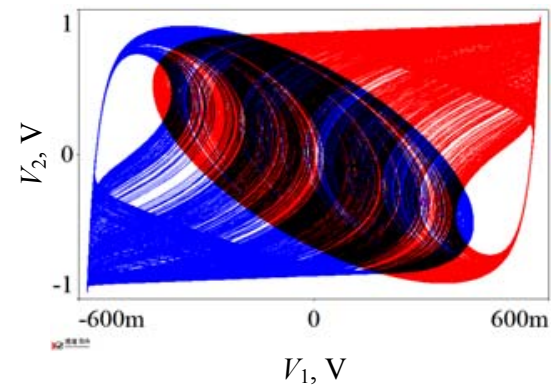

(d)

FIGURE VI. THE PHASE PORTRAITS IN V1 - V2 PLANE WITH DIFFERENT R ACHIEVED BY CIRCUIT SIMULATIONS. (A) $\mathrm{R}=$ $2.9 \mathrm{~K} \Omega$; (B) $\mathrm{R}=3.1 \mathrm{~K} \Omega$; (C) $\mathrm{R}=3.15 \mathrm{~K} \Omega$; (D) $\mathrm{R}=3.25 \mathrm{~K} \Omega$.
In this article, a new smooth inductorless Chua's circuit is presented on the basis of a second order Sallen-Key BPF, where the Chua's diode is achieved through active diode pair. The results from numerical simulations and circuit simulations confirm that this circuit can exhibit rich physical phenomenon of coexisting periodic attractors, coexisting single-scroll chaotic attractors, double-scroll chaotic attractor, periodic windows, boundary crisis and coexisting period-doubling bifurcation modes. The inductorless design method provides convenience for various potential engineering applications of chaos.

\section{REFERENCES}

[1] L. Fortuna, M. Frasca, M. G. Xibilia. Chua's circuit implementation: yesterday, today and tomorrow. Singapore: World Scientific, 2009.

[2] L. Zhu, M. H. Pan, and X. H. Qiao, "A chaotic circuit under a new classification framework of inductorless Chua's circuits", Circuit World. Vol. 45, pp. 208-220, November 2019.

[3] B. Carter, R. Mancini, Op amps for everyone. Mill Valley, USA: Elsevier Inc., 2009.

[4] B. C. Bao, H. G. Wu, L. Xu, M. Chen, and W. Hu, "Coexistence of multiple attractors in an active diode pair based Chua's circuit", International Journal of Bifurcation and Chaos. Vol. 28, pp. 1850019, February 2018.

[5] G. A. Leonov, N. V. Kuznetsov, "Hidden attractors in dynamical systems. From hidden oscillations in Hilbert-Kolmogorov, Aizerman, and Kalman problems to hidden chaotic attractor in Chua circuits", International Journal of Bifurcation and Chaos. Vol. 23, pp. 1330002, January 2013.

[6] A. Wolf, J. B. Swift, H. L. Swinney, and J. A. Vastano, "Determining Lyapunov exponents from a time series", Physica D: Nonlinear Phenomena. Vol. 16, pp. 285-317, 1985. 